\title{
A Self-Tuning PID Control Method for Multi-Input-Multi- Output Nonlinear Systems
}

\author{
Barış Bıdıklı \\ Department of Mechatronics Engineering, School of Engineering and Architecture, Izmir Katip Çelebi University, İzmir, Turkey
}

Cite this article as: B. Bıdıkl, "A Self-Tuning PID Control Method for Multi-Input-Multi-Output Nonlinear Systems", Electrica, vol. 18, no: 2, pp. 218-226, 2018.

\begin{abstract}
In this study, an artificial neural network (ANN) model-based self-tuning PID control method is proposed for the control of multi-input-multi-output (MIMO) nonlinear systems. A single layer, feed-forward ANN structure is trained via input and output data randomly collected from the system and classified as learning, test, and validation data to obtain the system model. The obtained model is utilized in an adaptive PID control scheme in conjunction with two different optimization methods for PID tuning and control. Using this scheme, PID parameters can be tuned to their optimum values and the system can be controlled simultaneously. The performance of the proposed method is demonstrated via experimental studies.
\end{abstract}

Keywords: Multi-input-multi-output nonlinear systems, proportional-integral-derivative tuning, proportional-integral-derivative control

\section{Corresponding Author: \\ Barış Bıdıklı}

\section{E-mail:}

baris.bidikli@ikc.edu.tr

Received: 15.01.2018

Accepted: 18.04 .2018

(c) Copyright 2018 by Electrica

Available online at

http://electrica.istanbul.edu.tr

DOI: 10.26650/electrica.2018.79181

\section{Introduction}

Despite significant improvements in the field of control, proportional-integral-derivative (PID) type controllers still preserve their popularity and importance in scientific and industrial applications. Their simple design procedure, as well as their robustness and ability to control many different types of linear and nonlinear systems efficiently, can be considered as the main reasons for this. In the literature on this area of research, it can be seen that PID controllers are still widely preferred for the control of many different types of systems in the last decade.

An improved PID switching control strategy was proposed for the closed-loop control system of an artificial pancreas to cope with Type 1 Diabetes in [1]. A deduced model based self-tuning PID control strategy was proposed for implementing a motion control system that stabilizes the two-wheeled vehicle and follows the desired motion commands in [2]. Velocity and orientation tracking control of a nonholonomic mobile robot was provided via an adaptive controller of nonlinear PID-based neural networks in [3]. A direct current (DC) motor speed control was provided via a particle swarm optimization supported by an optimal PID controller in [4]. Adaptive fuzzy PID controllers were utilized for the speed control of a DC motor in [5-6]. The overall performance of the electro-hydraulic position servo system of a servo hydraulic press was improved by means of a fuzzy PID control method while a hybrid fuzzy-PID controller was utilized to provide the position control of a hydraulic actuation system in [7-8]. A PID-type fuzzy adaptive controller was utilized for the control of an expert heating, ventiliating and air-conditioning system having two different zones with variable flow-rate in [9]. A real-time particle-swarm-optimization-based PID controlller was designed for the levitated balancing and propulsive positioning of a magnetic-levitation transportation system in [10]. A PID controller was utilized for position and orientation control, as well as for the attitude and position trackings of unmanned quadrotors in [11, 12]. In addition to these, a gain-scheduled PID control based active fault-tolerant technique was developed and applied to a similar vehicle in [13]. PID and linear quadratic regulator based optimal 
control techniques were proposed to control the nonlinear inverted pendulum dynamical system in $[14,15]$. A combination of an industrial linear PID controller and a neural compensator was used to minimize steady-state error with respect to uncertainties in robot control in [16]. An interval type-2 fuzzy PID controller was utilized for the trajectory tracking task of a 5 degree-of-freedom (DOF) redundant robot manipulator while the global finite-time regulation of robotic manipulators was provided via a nonlinear PID controller in [17, 18]. PID control was proposed as a solution for the feedback control problem of fully-constrained cable driven parallel manipulators in [19]. A novel fuzzy-PID approach based on feedforward control was utilized to regulate the oxygen excess ratio of the proton exchange membrane fuel cell system, while another feedforward PID control system was proposed to enhance the practical positioning performance of a piezo-actuated flexible 2-DOF micromanipulator integrated with a pair of modified differential lever displacement amplifiers in [20, 21]. An adaptive robust hybrid PID and sliding control optimized by multi-objective genetic algorithm optimization was presented to control a biped robot walking in the lateral plane on a slope in [22]. The aim was to control the blade pitch angle of a wind turbine at different wind speeds and to hold the output power stable at a specific set point by utilizing fuzzy logic PID controller in [23]. A new control method of combining a novel positive temperature coefficient material with PID control algorithm was proposed in [24]. A PID-based active vibration control system of an aluminum plate was designed, developed and experimentally verified in [25]. A new control design for an autonomous underwater vehicle was presented in [26]. In order to do this the researchers utilized a nonlinear auto-regressive moving-average exogeneous model of the vehicle and the PID controller.

As can be seen from the given examples PID and PID-based controllers can be used to control many different types of systems in a wide variety of areas, ranging from daily life to biomedical applications, from military applications to transportation. At this point it should also be noted that the mentioned studies are only a selection of the numerous PID control studies available in the literature. Although, the PID controller has been widely used for the control of many different types of systems, optimum tuning of the PID parameters (i.e., proportional, integral and derivative gains), which can be considered as the main issue of the PID control process, still remains a problem. PID parameters that are not tuned properly negatively affect the control performance. In some cases the stability of the system may be negatively affected by the tuning. This problem can be observed in most of the aforementioned studies and other PID control applications. As a result of this, proposing tuning methods for PID parameters has become a popular topic in the research area of the control. Detailed surveys on this topic can be found in $[27,28]$. This topic has also preserved its popularity in the last decade. A new robust PID tuning method for the op-

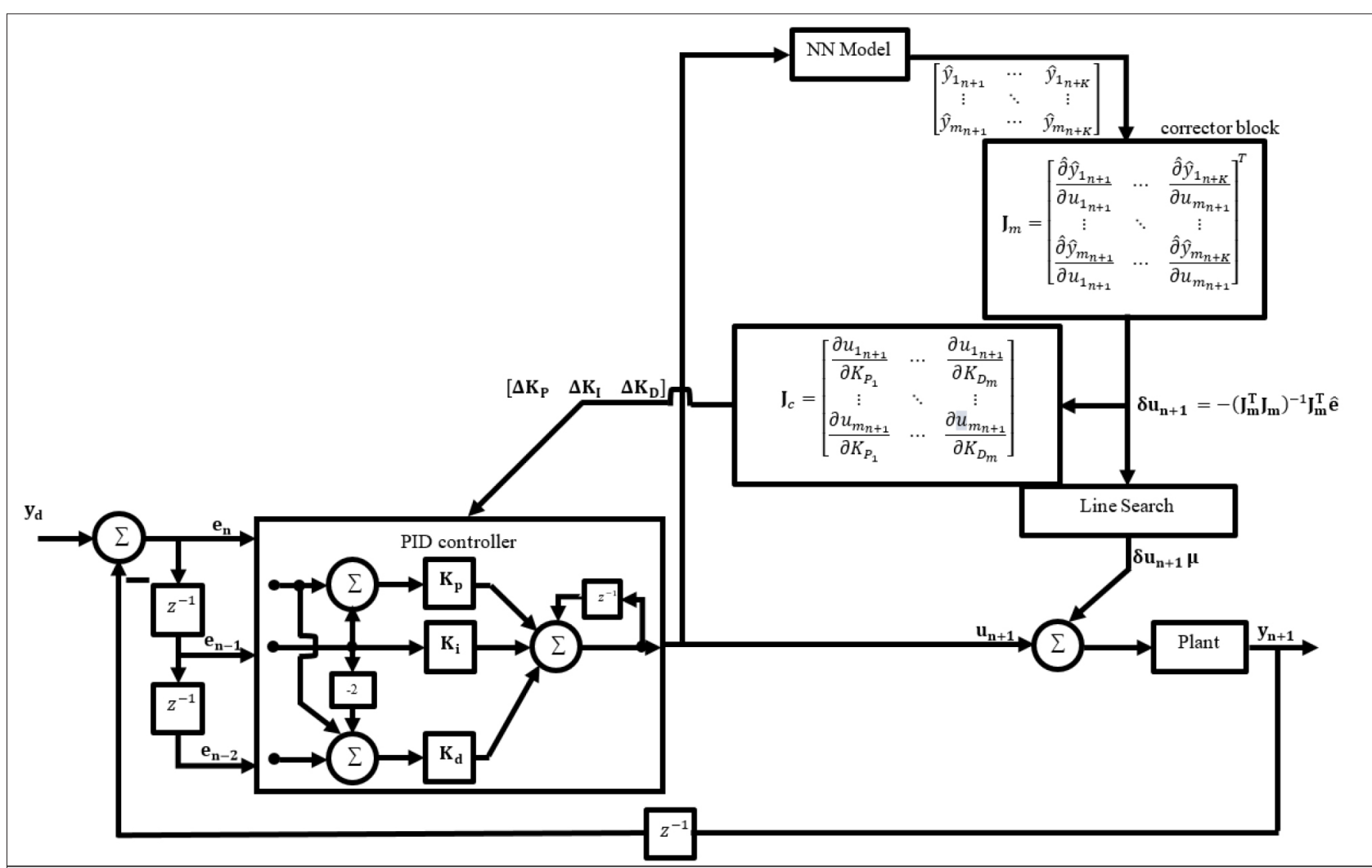

Figure 1. ANN model based PID tuning and control scheme 
timal closed-loop performance with specified gain and phase margins based on nonlinear optimization was developed in [29]. A stochastic, multi-parameters, divergence optimization method for the auto-tuning of PID controllers according to a fractional-order reference method was presented in [30]. Gain and phase margin specifications of the inner and outer loop based internal model control plus PID tuning procedure was proposed in [31]. A new model reduction method and an explicit PID tuning rule for the purpose of PID auto-tuning based on a fractional order plus time delay model are presented in [32]. A Newmark method based PID control rule was proposed for the active vibration control of multi-DOF flexible systems in [33]. One could easily add to these examples. However, the aforementioned studies are enough to clarify these issues. PID control can be considered to be a feasible solution for the control of nonlinear systems, and combining the PID control with an appropriate gain tuning method increases this feasibility.

One of the most suitable approaches was proposed to cope with these issues [34]. In the mentioned study, a PID based control scheme that can be used for the control of single-input-single-output nonlinear systems was proposed. In this scheme, control was provided via a digital PID controller combined with a nonlinear auto-regressive exogeneous (NARX) system model of the controlled system. Using this structure PID gains were adjusted online during the control process by utilizing the NARX model of the system. The control of the system was provided by utilizing a correction term that was computed by using the NARX model during the control gain adjusting process. According to the given structure when the gains reach their optimum values, the NARX model is deactived and the control process is continued with the classical PID approach. The structure of the proposed scheme provides an opportunity for the controller to adapt the changes in the system and/or enviromental factors during the control process. The proposed method is a suitable method when considering its ability to control nonlinear systems. However, most of the nonlinear systems have more than one input and output. As a result of this they are modeled as multi-input-multi-output (MIMO) systems. It is not possible to apply the above-mentioned method to these types of system with its current structure. This situation can be seen as the possible weakness of the proposed method. Moreover, performance of the proposed method was not experimentally verified in [34]. The main purpose of this study is to eliminate all of the above-mentioned deficiencies and to make the proposed method usable for a broader class of nonlinear systems. The system is brought into the appropriate structure for MIMO nonlinear systems. Since it is necessary to make a lot of changes to the structure of the system it is a challenging task. Performance of the newly proposed method is experimentally verified by utilizing from it to provide the joint space control of a rigid link 2-DOF robot manipulator. This experimental verification can be seen as another contribution of this study.

The rest of the paper is organized as follows. In section 2, the structure of the proposed artificial neural network (ANN) mod- el based PID tuning and control scheme is presented and explained in a detailed manner. In section 3, the architecture and the learning algorithm of the utilized ANN is summarized. In section 4, the performance of the proposed method is demonstrated via experimental studies Finally, conclusions are given in section 5 .

\section{Artificial Neural Network (ANN) Model Based PID Tuning and Control Method}

The structure of the proposed scheme is shown in Figure 1. As mentioned in the previous section, this structure is the rearranged version of the structure given in accordance with the structures of MIMO systems [34]. Jacobian calculation block used for computing the correction term of the control signal and another Jacobian calculation block used for PID tuning are rearranged according to MIMO systems having $m$ inputs and $m$ outputs. Moreover, the structure of the PID controller, ANN model and Line Search blocks were made available for MIMO systems. As a result of this, PID parameters denoted by $\mathbf{K}_{\mathbf{p}^{\prime}} \mathbf{K}_{\mathbf{l}^{\prime}}$ and $\mathbf{K}_{\mathbf{D}^{\prime}}$ became $m \times m$ diagonal matrices. In this structure desired trajectory, outputs of the ANN model, the plant and the error are denoted by $\mathbf{y}_{\mathrm{d}^{\prime}}, \hat{\mathbf{y}}, \mathbf{y}$, and $\mathbf{e}_{\mathrm{n}} \in \mathbb{R}^{\mathrm{m}}$ respectively. The time index is represented by $n \in \mathbb{R}$ while the control input and its corrections are denoted by, $U_{n+1}, \delta u_{n+1} \mu \in \mathbb{R}^{m}$, respectively. As a result of these structure and dimension changes, mathematical analysis and design must be completely rearranged.

The control input is updated according to the update rule of the digital PID controller given as

$$
\begin{aligned}
\mathbf{u}_{n+1}=\mathbf{u}_{n} & +\mathbf{K}_{P}\left(\mathbf{e}_{n}-\mathbf{e}_{n-1}\right)+\mathbf{K}_{I} \mathbf{e}_{n} \\
& +\mathbf{K}_{D}\left(\mathbf{e}_{n}-2 \mathbf{e}_{n-1}+\mathbf{e}_{n-2}\right)
\end{aligned}
$$

Tuning the PID parameters starting from zero to their proper values is the main purpose of the proposed structure. The $K-$ step ahead prediction of the plant's behavior is employed for this purpose. This prediction is produced by applying the control input given in (1) to the ANN model of the plant $K$ times. It is attempted to minimize the following objective function for each output of the system by updating the PID parameters based on these predictions

$$
\begin{aligned}
J_{i}\left(u_{1_{n+1}}, u_{2_{n+1}}, \cdots, u_{m_{n+1}}\right) & \\
& =\frac{1}{2}\left[\sum _ { k = 1 } ^ { K } \left(y_{d_{i_{n+k}}}\right.\right. \\
& \left.-\hat{y}_{i_{n+k}}\right)^{2} \\
& \left.+\sum_{\ell=1}^{m} \lambda_{\ell}\left(u_{\ell_{n+1}}-u_{\ell}\right)^{2}\right]
\end{aligned}
$$


where the subscript $i=1, \ldots, m$ and the diagonal matrix whose diagonal elements are equal to penalty terms and the prediction horizon are denoted by $\lambda \in \mathbb{R}^{\mathrm{m} \times \mathrm{m}}$ and $K \in \mathbb{R}$ , respectively. The Levenberg-Marquardt (LM) rule is utilized as the minimization algorithm in the proposed structure and this rule realizes the minimization according to the following equation

$$
\left[\begin{array}{c}
\mathbf{K}_{\mathbf{p}_{\text {new }}} \\
\mathbf{K}_{\mathbf{I}_{\text {new }}} \\
\mathbf{K}_{\mathbf{D}_{\text {new }}}
\end{array}\right]=\left[\begin{array}{c}
\mathbf{K}_{\mathbf{p}_{\text {old }}} \\
\mathbf{K}_{\mathbf{I}_{\text {old }}} \\
\mathbf{K}_{\mathbf{D}_{\text {old }}}
\end{array}\right]-\left(\mathbf{J}^{\mathbf{T}} \mathbf{J}+\mu_{g} \mathbf{I}_{3 m}\right)^{-1} \mathbf{J}^{\mathbf{T}} \hat{\boldsymbol{e}}
$$

where $\mathbf{I}_{3 m} \in \mathbb{R}^{3 m \times 3 m}$ denotes the standard identity matrix, while the parameter that provides the transition between the steepest-descent and the Gauss-Newton algorithms is denoted by $\mu_{g} \in \mathbb{R}$. The Jacobian matrix represented by $\mathbf{J} \in$ $\mathbb{R}^{(m K+1) \times 3 m}$ is defined as

$$
\mathbf{J} \triangleq-\left[\begin{array}{cccc}
\frac{\partial \hat{y}_{1_{n+1}}}{\partial K_{P_{1}}} & \frac{\partial \hat{y}_{1_{n+1}}}{\partial K_{I_{1}}} & \cdots & \frac{\partial \hat{y}_{1_{n+1}}}{\partial K_{D_{m}}} \\
\vdots & \vdots & \ddots & \vdots \\
\frac{\partial \hat{y}_{1_{n+K}}}{\partial K_{P_{1}}} & \frac{\partial \hat{y}_{1_{n+K}}}{\partial K_{I_{1}}} & \ddots & \frac{\partial \hat{y}_{1_{n+K}}}{\partial K_{D_{m}}} \\
\frac{\partial \hat{y}_{2_{n+1}}}{\partial K_{P_{1}}} & \frac{\partial \hat{y}_{2_{n+1}}}{\partial K_{I_{1}}} & \ddots & \frac{\partial \hat{y}_{2_{n+1}}}{\partial K_{D_{m}}} \\
\vdots & \vdots & \vdots & \vdots \\
\frac{\partial \hat{y}_{m_{n+K}}}{\partial K_{P_{1}}} & \frac{\partial \hat{y}_{m_{n+K}}}{\partial K_{I_{1}}} & \ddots & \frac{\partial \hat{y}_{m_{n+K}}}{\partial K_{D_{m}}} \\
\sqrt{\lambda_{1} \frac{\partial u_{1_{n+1}}}{\partial K_{P_{1}}}} & \sqrt{\lambda_{1}} \frac{\partial u_{1_{n+1}}}{\partial K_{I_{1}}} & \cdots & \sqrt{\lambda_{m}} \frac{\partial u_{m_{n+1}}}{\partial K_{D_{m}}}
\end{array}\right]
$$

and $\hat{\mathbf{e}} \in \mathbb{R}^{(m K+1) \times m}$ that contains the prediction errors and the input related optimization terms is defined as

$$
\begin{aligned}
& \hat{\mathbf{e}} \\
& \triangleq-\left[\begin{array}{ccc}
\tilde{y}_{1_{n+1}}-\hat{y}_{1_{n+1}} & \cdots & \tilde{y}_{1_{n+1}}-\hat{y}_{1_{n+1}} \\
\vdots & \ddots & \vdots \\
\tilde{y}_{m_{n+K}}-\hat{y}_{m_{n+K}} & \ddots & \tilde{y}_{m_{n+K}}-\hat{y}_{m_{n+K}} \\
\sqrt{\lambda_{1}}\left(u_{1_{n+1}}-u_{1_{n}}\right) & \cdots & \sqrt{\lambda_{m}}\left(u_{m_{n+1}}-u_{m_{n}}\right)
\end{array}\right]
\end{aligned}
$$

The aim is to iteratively provide optimum tuning of the PID parameters during the control process according to the given structure and algorithms. In addition, obtaining an efficient control performance during the transient-state, increasing robustness against possible disturbances and adaptivity of the proposed method are other aims. All of these situations are realized by utilizing correction term. This term compensates the deficiency of the control input when it cannot be provided by the controller, otherwise it disables. Mathematically, it can be said that the corrector block tries to minimize the elements of the objective functions' vector denoted by $J i$ with respect to $\delta_{u_{\ell_{n+1}}}$ (i.e., correction term of the $\ell^{t h}$ control input that specifically affects the $i^{\text {th }}$ output) based on the second-order Taylor approximation of this element given as

$$
\begin{aligned}
& J_{i}\left(u_{1_{n+1}}+\delta_{u_{1_{n+1}}}, u_{2_{n+1}}+\delta_{u_{2_{n+1}}}, \cdots, u_{m_{n+1}}\right. \\
& \left.+\delta_{u_{m_{n+1}}}\right) \\
& \cong J_{i}\left(u_{1_{n+1}}, u_{2_{n+1}}, \cdots, u_{m_{n+1}}\right) \\
& +\sum_{\ell=1}^{m} \frac{\partial J_{i}\left(u_{1_{n+1}}, u_{2_{n+1}}, \cdots, u_{m_{n+1}}\right)}{\partial u_{\ell_{n+1}}} \delta_{u_{\ell_{n}+1}} \\
& +\frac{1}{2}\left[\sum_{\ell=1}^{m} \frac{\partial^{2} J_{i}\left(u_{1_{n+1}}, u_{2_{n+1}}, \cdots, u_{m_{n+1}}\right)}{\partial u_{\ell_{n+1}}^{2}}\left(\delta u_{\ell_{n+1}}\right)^{2}\right]
\end{aligned}
$$

Values of $\delta_{u_{\ell_{n+1}}}$ terms that minimize the objective function can be found by taking the derivative of (6) with respect to this term and equalizing the result to zero as

$$
\begin{aligned}
& \delta_{u_{\ell_{n+1}}} \\
& =-\frac{\frac{\partial J_{i}\left(u_{1_{n+1}}, u_{2_{n+1}}, \cdots, u_{m_{n+1}}\right)}{\partial u_{\ell_{n+1}}}}{\frac{\partial^{2} J_{i}\left(u_{1_{n+1}}, u_{2_{n+1}}, \cdots, u_{m_{n+1}}\right)}{\partial^{2} u_{\ell_{n+1}}^{2}}} .
\end{aligned}
$$

The term in (7), corresponds to the Newton direction and this provides a quadratic convergence to the local minimum when the Hessian terms in the Taylor expansion are positive and the higher order terms are negligible [35]. The following $(K m+1) \times m$ matrix proposed by a Jacobian approximation is utilized to avoid the time consuming calculation of second-order derivatives

$\mathbf{J}_{\mathbf{m}}=-\left[\begin{array}{ccc}\frac{\partial \hat{y}_{1_{n+1}}}{\partial u_{1_{n+1}}} & \cdots & \frac{\partial \hat{y}_{1_{n+1}}}{\partial u_{m_{n+1}}} \\ \vdots & \ddots & \vdots \\ \frac{\partial \hat{y}_{m_{n+K}}}{\partial u_{1_{n+1}}} & \ddots & \frac{\partial \hat{y}_{m_{n+K}}}{\partial u_{m_{n+1}}} \\ \sqrt{\lambda_{1}} & \cdots & \sqrt{\lambda_{m}}\end{array}\right]$.

The gradient term can exactly be represented and the Hessian term can approximately be represented via the matrix given in (8) as

$$
\begin{aligned}
& \mathbf{D}_{\mathbf{u}_{n+1}}^{1} \mathbf{J}\left(\mathbf{u}_{n+1}\right)=2 \mathbf{J}_{\mathbf{m}}^{\mathrm{T}} \hat{\mathbf{e}} \\
& \mathbf{D}_{\mathbf{u}_{n+1}}^{2} \mathbf{J}\left(\mathbf{u}_{n+1}\right) \cong 2 \mathbf{J}_{m}^{T} \mathbf{J}_{m}
\end{aligned}
$$

where the operator $\mathbf{D}_{d}^{\mathbf{o}}$ represents the $o^{\text {th }}$ order derivative of a vector or a matrix with respect to the variable $d$. The vector of the correction terms can be computed as 
$\boldsymbol{\delta}_{\mathbf{u}_{n+1}}=-\left(\mathbf{J}_{m}^{T} \mathbf{J}_{m}\right)^{-1} \mathbf{J}_{m}^{T} \hat{\mathbf{e}}$

where (7) and (9) were utilized. From (10) it is clear that we only need the first order derivatives. As demonstrated in Figure 1, it is possible to express the Jacobian matrix (4) as a multiplication of two matrices via the chain rule as $\mathbf{J}=\mathbf{J}_{m} \mathbf{J}_{C^{\prime}}$ the matrix denoted by $\mathbf{J}_{m}$ being one of these matrices, with the other matrix denoted by $\mathbf{J}_{C} \in \mathbb{R}^{\mathrm{m} \times 3 \mathrm{~m}}$ being expressed in terms of errors as

$\mathbf{J}_{c}=\left[\begin{array}{ccc}\frac{\partial u_{1_{n+1}}}{\partial K_{P_{1}}} & \cdots & \frac{\partial u_{1_{n+1}}}{\partial K_{D_{m}}} \\ \vdots & \ddots & \vdots \\ \frac{\partial u_{m_{n+1}}}{\partial K_{P_{1}}} & \cdots & \frac{\partial u_{m_{n+1}}}{\partial K_{D_{m}}}\end{array}\right]$

So it is clearly seen that both of these matrices have crucial roles in the proposed structure since they are utilized to compute the Jacobian matrix that is used to tune the PID parameters. Moreover, $\mathbf{J}_{m}$ has an another critical role. It preserves the efficiency of the control by providing the correction terms. The minimization of the objective function can be realized via the the vector of optimum step lengths $\boldsymbol{\mu} \in \mathbb{R}^{\boldsymbol{m}}$ computed in the last block namely as line search. Since every term except the step length was determined before this point, the general problem transforms into multi-dimensional optimization problem according to this step length. It can be solved via any method whose structure is feasible for this purpose. Gauss-Newton algorithm is preferred to cope with this issue in this study [36]. Additionaly, ANN model of the MIMO nonlinear system is utilized for the calculation of the Jacobian matrix. A detailed attempt at explaining the modeling procedure is given in the following section.

\section{Artificial Neural Network (ANN) Modeling, Prediction and Jacobian Calculation}

From the given structure it is clear that its performance is directly related to the representation capability of the ANN model of the plant. Jacobian matrix in (4) is based on the predictions of the ANN model and this matrix is utilized both for tuning of the PID parameters and for computation of the correction terms. It can easily be considered that the ANN model is the most important part of the proposed method. In this section, ANN modeling, prediction and Jacobian calculation used in this study are examined in detail.

The mathematical expression of an ANN model of a MIMO nonlinear system is given as

$$
\begin{gathered}
Y_{r n}=f\left(u_{1_{n}}, \cdots, u_{1\left(n-n_{u}\right)}, \cdots, u_{m\left(n-n_{u}\right)}, y_{1(n-1)},\right. \\
\left.\cdots, y_{1\left(n-n_{y}\right)}, \cdots, y_{m\left(n-n_{y}\right)}\right)
\end{gathered}
$$

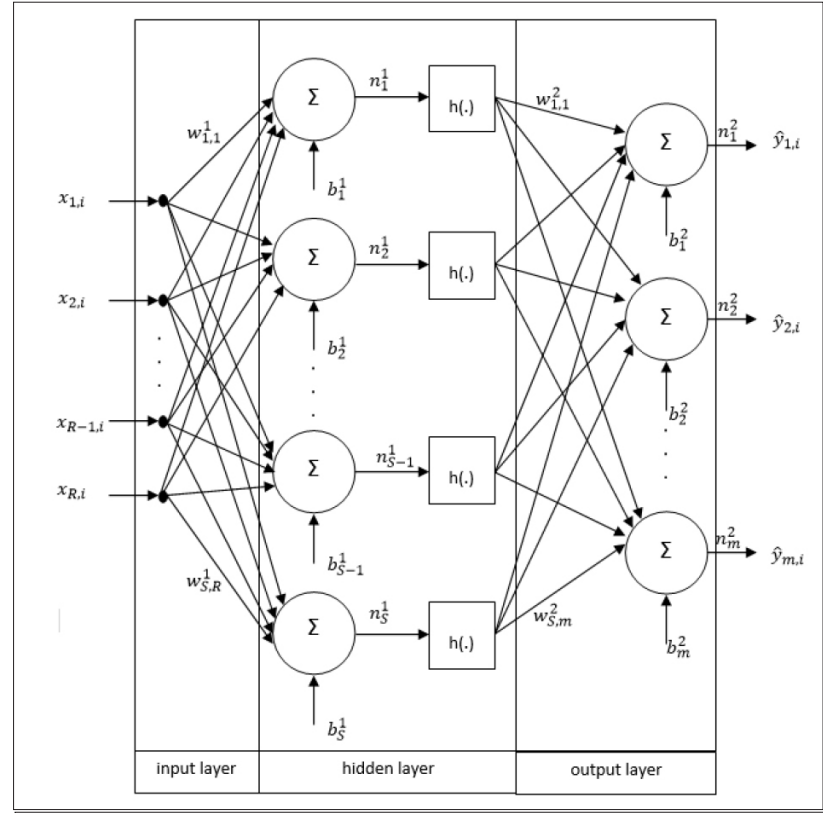

Figure 2. ANN Structure

where $u_{r n} \in \mathbb{R}$ represents the $n^{\text {th }}$ value of the $r^{t h}$ input while the $n^{\text {th }}$ value of the $r^{t h}$ output is represented by $Y_{n_{n}} \in \mathbb{R}$. The number of the past values of inputs of the ANN model are denoted by $n_{u}$ and $n_{y} \in \mathbb{R}$, respectively. The uncertain function denoted by $f(\cdot)$ can be obtained by applying the learning data to ANN according to the relation mathematically expressed as

T

$=\left\{u_{1 k}, \cdots, u_{1\left(k-n_{u}\right)}, y_{1(k-1)}, \cdots, y_{1\left(k-n_{y}\right)}\right.$,

$\left.y_{m\left(k-n_{y}\right)}, \cdots ; y_{1 k}, \cdots, y_{m k}\right\}_{k=n}^{k=n+N}$

$=\left\{x_{k}, y_{k}\right\}_{k=1}^{k=N}$

where $x_{k} \in \mathbb{R}_{\mathrm{X}} \subseteq \mathbb{R}^{\left[r n_{u}+m\left(n_{y}+1\right)\right]}$ represents the input data point from the input space while an appropriate output value is denoted by $y_{k} \in \mathbb{R}_{\mathrm{y}} \subseteq y_{k} \in \mathbb{R}_{\mathrm{y}} \subseteq$. Obtaining a model that represents the relation between input and output data points is the main aim. Learning data group $T$ is used to obtain the approximate behavior of the system.

Single layer, feedforward, MIMO ANN structure used in this study is given in Figure 2.

In this structure the numerical value of $R \in \mathbb{R}$ is given as $R=r n_{u}+m\left(n_{y}+1\right)$ while weights from $i^{t h}$ input to $j^{t h}$ neuron cell and from $j^{\text {th }}$ neuron cell to $k^{\text {th }}$ output are denoted by $\omega_{j, i}^{1}$ and $\omega_{j, k}^{2}$, respectively. Bias values from $j t h$ neuron cell to hidden layer and from output layer to $k^{\text {th }}$ output are denoted by $b_{1}^{j}$ and $b_{2}^{k}$, respectively. These values are adjustable parameters of the ANN updated in every single iteration until their optimum values are obtained. 
The input-output relationship of an ANN model is expressed as $\hat{y}_{k n}=\sum_{k=1}^{m}\left(\sum_{j=1}^{S} \omega_{k, j}^{2} h\left(d_{j, n}\right)+b_{k}^{2}\right)$

where $h(x) \in \mathbb{R}$ represents the sigmoidal activation function expressed as $h(x)=\frac{e^{x}-e^{-x}}{e^{x}+e^{-x}}$. Number of neurons in the hidden layer are denoted by $S \in \mathbb{R}$ and $d_{j, n} \in \mathbb{R}$ is computed as

$d_{j, n}$

$=\sum_{k=1}^{r}\left(\sum_{i=0}^{n_{u}} \omega_{j, i}^{1} u_{k(n-i)}\right)$

$+\sum_{l=1}^{m}\left(\sum_{i=1}^{n_{y}} \omega_{j, n_{u}+i+1}^{1} y_{i(n-i)}\right)+b_{j}^{1}$

The following objective function is minimized by updating the adjustable parameters in every single iteration

$F=\sum_{l=1}^{m} \sum_{k}^{N}\left(y_{l k}-\hat{y}_{l k}\right)^{2}$

The LM rule is utilized in every iteration to optimize the adjustable parameters as

$$
\begin{aligned}
\boldsymbol{\Theta}^{\text {new }}=\boldsymbol{\Theta}^{\text {old }}- & \left(\mathbf{J}_{A N N}^{T} \mathbf{J}_{A N N}\right. \\
& \left.+\boldsymbol{\mu}_{A N N} \mathbf{I}_{R}\right)^{-1} \mathbf{J}_{A N N}^{T} \mathbf{e}_{A N N}
\end{aligned}
$$

where $\mathbf{e}_{A N N} \in \mathbb{R}^{\mathrm{MN} \times \mathrm{R}}$ represents the vector that contains learning errors and defined as

$$
\begin{aligned}
& \mathbf{e}_{A N N} \\
& =\left[\begin{array}{lllll}
y_{11}-\hat{y}_{11} & \cdots & y_{1 N}-\hat{y}_{1 N} & \cdots & y_{M N}-\hat{y}_{M N}
\end{array}\right]
\end{aligned}
$$

$\boldsymbol{\Theta} \in \mathbb{R}^{\mathrm{R}}$ is a vector of weights and biases

$$
\begin{aligned}
& \boldsymbol{\Theta}=\left[\begin{array}{llllll}
\omega_{1,1}^{1} & \cdots & \omega_{s, n_{u}+N_{y}+1}^{1} & b_{1}^{1} & \cdots & b_{s}^{1} \\
\omega_{1,1}^{2} & \cdots & \omega_{m, s}^{2} & b_{1}^{2} & \cdots & b_{m}^{2}
\end{array}\right]^{\mathrm{T}} \\
&= {\left[\begin{array}{llll}
\theta_{1} & \cdots & \theta_{R}
\end{array}\right]^{\mathrm{T}} }
\end{aligned}
$$

and $\mathbf{J}_{A N N} \in \mathbb{R}^{\mathrm{MN} \times \mathrm{R}}$ is a Jacobian matrix whose structure is given as

$$
\mathbf{J}_{A N N}=\left[\begin{array}{ccc}
\frac{\partial e_{A N N_{11}}}{\partial \theta_{1}} & \cdots & \frac{\partial e_{A N N_{11}}}{\partial \theta_{R}} \\
\vdots & \ddots & \vdots \\
\frac{\partial e_{A N N_{M N}}}{\partial \theta_{1}} & \cdots & \frac{\partial e_{A N N_{M N}}}{\partial \theta_{R}}
\end{array}\right]
$$

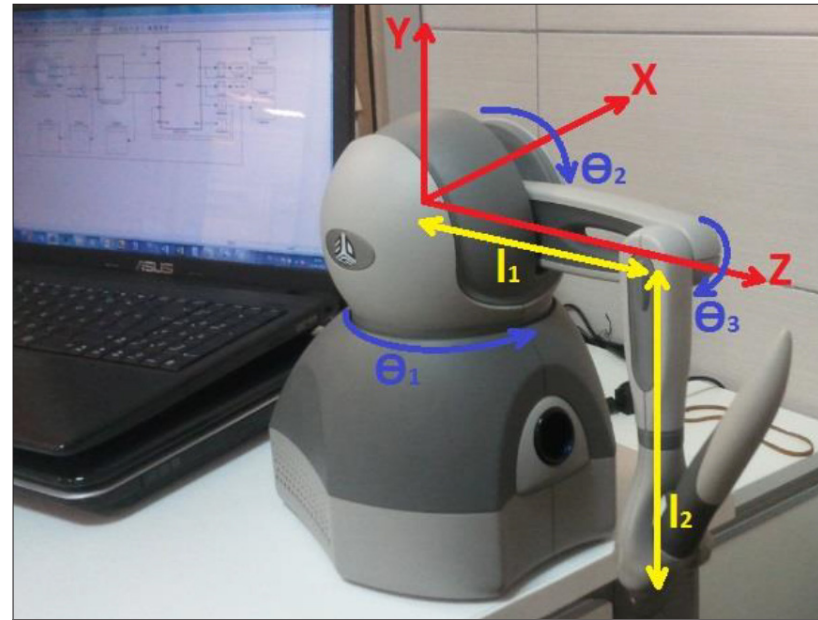

Figure 3. Phantom OMNI Haptic Device

The ANN model can be obtained by utilizing the given equations and realizing the necessary number of iterations until the value of the objective function in (15) reaches the desired level. The future behavior of the plant can be predicted by utilizing (14), after the ANN model has been obtained.

\section{Algorithm of the ANN Model-Based PID Tuning and Control}

After the necessary arrangements are realized on the structure and mathematical design, the algorithm given in [34] can be used directly. The steps of the proposed algorithm can be itemized as follows:

Step 1: The minimum and maximum input values $u_{\min }$ and $u_{\max }$ that can be applied to the plant are determined. Then the modeling data is collected from the system by applying random inputs from the interval given as $\left[u_{\text {min' }}, u_{\text {max }}\right]$ and measuring the outputs of the plant.

Step 2: A set of training data is formed after the $n_{u}$ and $n_{y}$ values of the ANN model (13) are determined. Then, the set of input and output values are normalized to the interval .

Step 3: From the collected $N$ data from the plant, data pairs are randomly selected as the learning data for the ANN model, the remaining data are used as test and validation data pairs.

Step 4: PID tuning and control are realized by applying the following algorithm at each iteration:

- The corresponding control input $\mathbf{u}_{n+1}$ is computed according to the measured error $\mathbf{e}_{n}$.

- The $K$-step future behavior of the system is predicted by utilizing the ANN model of the plant. To realize this prediction the $K$-step input sequence $\left[u_{n+1}, \cdots, u_{n+1}\right]$ is applied to the ANN model.

- The Jacobian matrix $\mathbf{J}_{m}$ is calculated by utilizing ANN model and then the correction term is determined by 


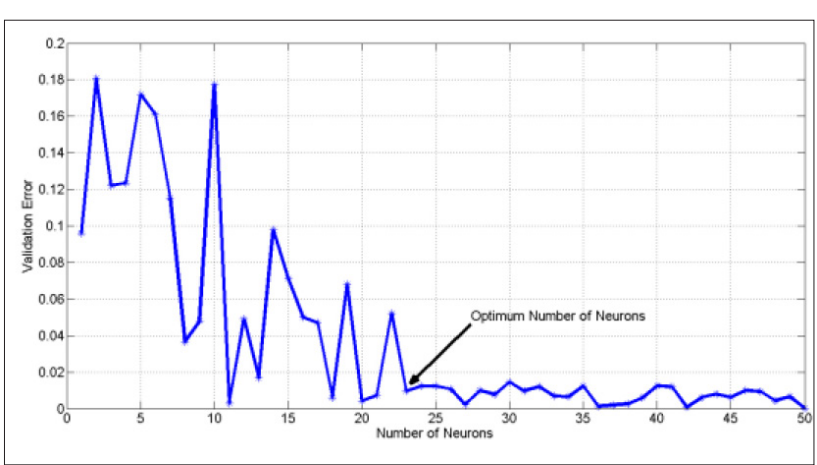

Figure 4. Selection of Number of Hidden Neurons

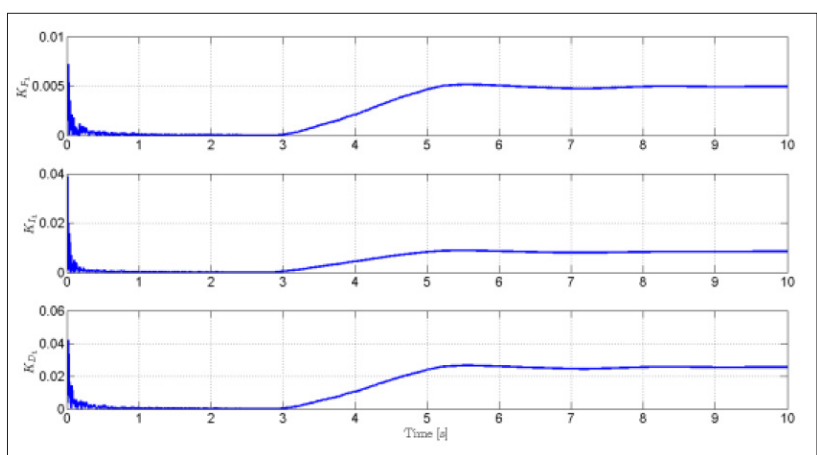

Figure 5. Control Gains for $\mathrm{u}_{1}(t)$

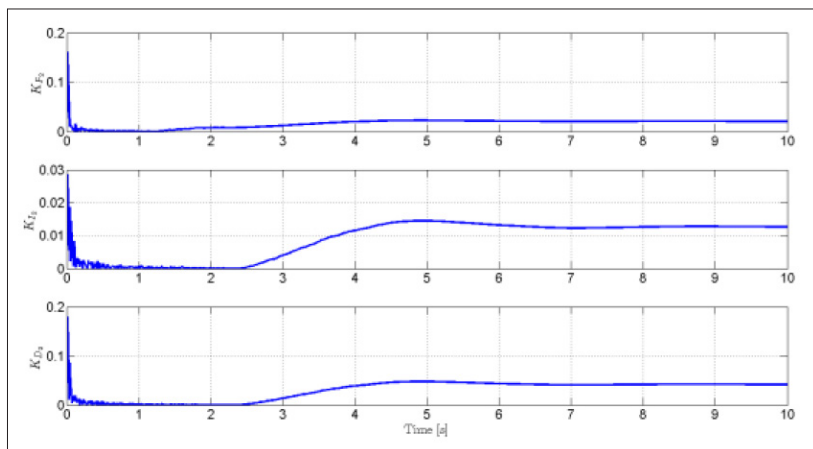

Figure 6. Control Gains for $\mathrm{u}_{2}(t)$

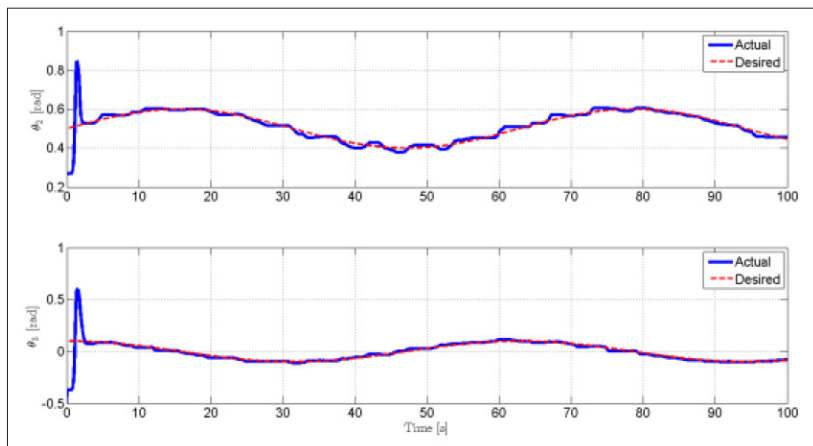

Figure 7. Actual (line) and Reference (dashed) Trajectories

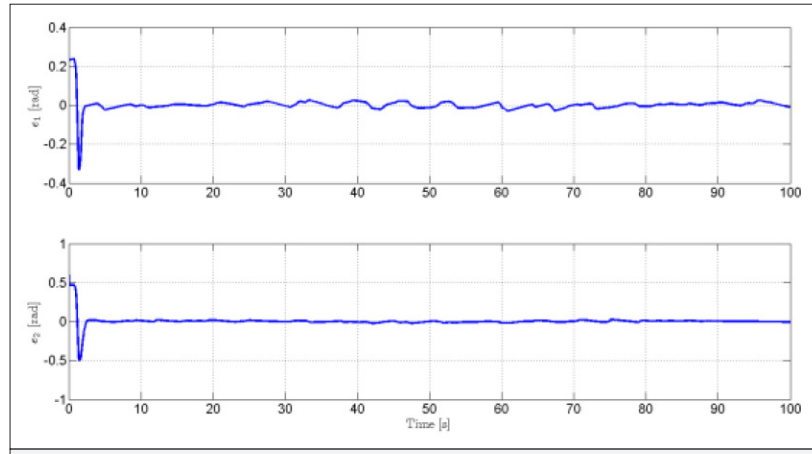

Figure 8. Link Tracking Errors
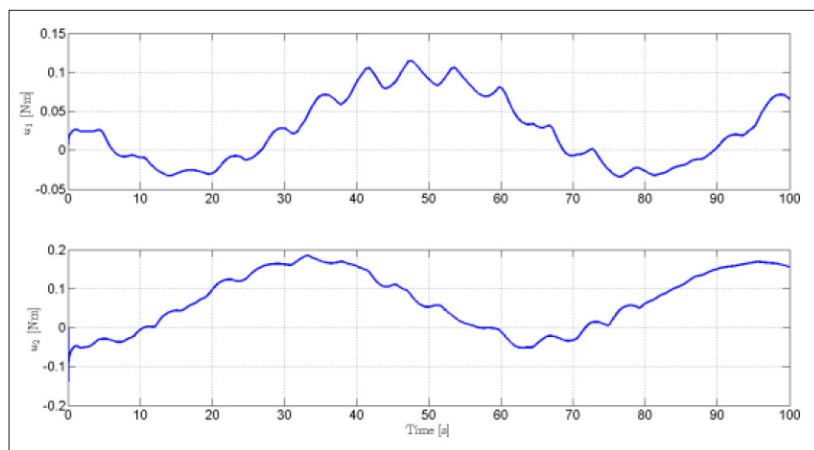

Figure 9. Control Inputs

utilizing both the ANN model and the calculated Jacobian matrix.

- The vector of optimum step sizes of the correction terms $\boldsymbol{\mu}$ is founded via the Gauss-Newton algorithm.

- Summation of the control input and the correction term (i.e., $\mathbf{u}_{n+1}+\boldsymbol{\delta} \mathbf{u}_{n+1} \boldsymbol{\mu}$ ) is applied to the system.

- The general Jacobian matrix (4) is calculated and the PID parameters are updated utilizing (3).

\section{Experimental Results}

Phantom OMNI haptic device shown in Figure 3 was used as an experimental setup. A 2-DOF rigid link robot manipulator structure was obtained by mechanically stopping the first joint whose angle was denoted by $\theta_{1} \in \mathbb{R}$. Joint space control application of the remaining links, whose angles were denoted by $\theta_{2}$ and $\theta_{3} \in \mathbb{R}$, respectively, was realized for the performance demonstration of the proposed method.

At this point it should be stated that each of the previously mentioned parameters has a crucial role for the proposed method and all of them should be selected appropriately. However, proposing different suitable methods for all of the selections is a hard and time consuming task. Moreover, it increases the computational complexity. Some of these parameters were fixed to some constant values by considering these issues. The prediction horizon and the matrix of weigthing factors were fixed to $K=10$ and $\lambda=$ $0.01 I_{2}$. Since, it was observed during the experiments that 5-step 
previous values of the inputs and the outputs provide the best validation error, the number of the past values of the inputs and the outputs were selected as $n_{u}$ and $n_{y}=5$, respectively.

The number of hidden neurons directly affects the modeling performance of the ANN. As a result of this, it can directly be related to the performance of the proposed method. Both of the selections that are less or more than the optimum value decrease the representation capability of the ANN model. The number of hidden neurons were selected to reduce the norm of the matrix containing the validation errors. To reach this purpose, ANN modeling was realized 1000 times for each number of neurons and the means of the norm values were marked on Figure 4. As it can be seen from Figure 4, any significant change was not observed in this value after 23 neurons. As a result of this, the optimum number of hidden neurons was selected as 23. At this point it should be stated that all of this process was applied to 1000 learning, 500 test and 500 validation data that were randomly selected from the collected input output data pairs from both links of the experimental system.

The control objective is to make $\theta_{2}(t)$ and $\theta_{3}(t)$ follow a sinusoidal desired trajectory chosen as

$\boldsymbol{\theta}_{r}(t)=\left[\begin{array}{c}0.5+0.1 \sin (0.1 t) \\ \cos (0.1 t)\end{array}\right](\mathrm{rad})$

The adjustment process of the control gains can be seen from Figure 5 and Figure 6 for $u_{1}(t)$ and $u_{2}(t)$, respectively. From these figures it can be seen that the control gains were tuned to their optimum values approximately in 4 seconds. Optimum values of control gains were obtained as $\mathrm{Kp}=\operatorname{diag}([0.005$, $0.02]), \mathrm{K}_{l}=\operatorname{diag}([0.008,0.013])$ and $\mathrm{K} D=\operatorname{diag}(0.025,0.04)$.

The desired and the actual trajectories are shown together in Figure 7, while the tracking errors and the control inputs are given in Figure 8 and Figure 9, respectively. From Figure 7 and Figure 8 , it can be seen that the control objective was met.

\section{Conclusion}

In this study, a self-tuning PID control scheme was proposed for the control of MIMO nonlinear systems. A number of optimization methods were used in conjuction with the ANN model of the system to ensure the self-tuning structure. First, the ANN model of the system was obtained via input and output data pairs collected from the system. These data pairs were randomly classified as learning, test and validation data and they were used to train a single layer, feedforward MIMO ANN structure. Then, the obtained ANN model was utilized for both PID tuning and control purposes. A vector of correction term computed via the Gauss-Newton algorithm was used to provide control until the PID parameters reach their optimum values. As a result of this, the control objective was met during the PID tuning process. Once the PID parameters reached their optimum values, the correction term was disabled and the pro- cess was continued with the classical PID control. Performance of the proposed method was demonstrated via a joint-space control application of a rigid link 2-DOF robot manipulator. In the experiments, it was observed that PID parameters were tuned to their optimum values in 4 seconds. However, from the actual and desired trajectories and the link tracking errors it was also seen that the control objective was met before this process and it continued after the optimum parameter values were provided. From these results, it can be said that all of the previously mentioned purposes were reached in the experimental studies.

Peer-review: Externally peer-reviewed.

Conflict of Interest: The authors have no conflicts of interest to declare.

Financial Disclosure: The authors declared that this study has received no financial support.

\section{References}

1. T. Marchetti, M. Barolo, L. Jovanovic, H. Zisser, D. E. Seborg, "An improved PID switching control strategy for Type 1 Diabetes", IEEE Trans on Biomed Eng, vol. 55, no. 3, pp. 857-865, 2008.

2. T. J. Ren, T. C. Chen and C. J. Chen, "Motion control for a twowheeled vehicle using a self-tuning PID controller", Control Engineering Practice, vol. 16, no. 3, pp. 365-375, 2008.

3. J. Ye, "Adaptive control of nonlinear PID-based analog neural networks for a nonholonomic mobile robot", Neurocomputing, vol. 71, no. 7-9, pp. 1561-1565, 2008.

4. B. Allaoua, B. Gasbaoui, B. Mebarki, "Setting up PID DC motor speed control alteration parameters using particle swarm optimization strategy", Leonaro Electronic J. of Practices and Technologies, no. 14, pp. 19-32, 2009.

5. R. Kandiban, R. Arulmozhiyal, "Design of adaptive fuzzy PID controller for speed control of BLDC motor", International Journal of Soft Computing and Engineering, vol. 2, no. 1, pp. 386-391, 2012.

6. U. K. Bansal and R. Narvey, "Speed control of DC motor using fuzzy PID controller", Advance in Electronic and Electric Engineering, vol. 3, no. 9, pp. 1209-1220, 2013.

7. J. M. Zheng, S. Zhao, S. Wei, "Application of self-tuning fuzzy PID controller for a SRM direct drive volume control hydraulic press", Control Engineering Practice, vol. 17, no. 12, pp. 1398-1404, 2009.

8. Ş. Çetin, A. V. Akkaya, "Simulation and hybrid fuzzy-PID control for positioning of a hydraulic system", Nonlinear Dynamics, vol. 61, no. 3, pp. 465-476, 2010.

9. S. Soyguder, M. Karakose, H. Alli, "Design and simulation of self-tuning PID-type fuzzy adaptive control for an expert HVAC system", Expert Systems with Applications, vol. 36, no. 3, pp. 45664573, 2009.

10. R. Wai, J. Lee, K. Chuang, "Real-time PID control strategy for maglev transportation system via particle swarm optimization", IEEE Transactions on Industrial Electronics, vol. 58, no. 2, pp. 629-646, 2011.

11. J. Li, Y. Li, "Dynamic analysis and PID control for a quadrotor", IEEE International Conference on Mechatronics and Automation China, 2011.

12. I. Sadeghzadeh, A. Mehta, A. Chamseddine, Y. Zhang, "Active fault tolerant control of a quadrotor UAV based on gainscheduled PID 
control", IEEE Canadian Conference on Electrical and Computer Engineering, Montreal, QC, Camada, 2012.

13. F. Goodarzi, D. Lee, T. Lee, "Geometric nonlinear PID control of a quadrotor UAV on SE(3)," European Control Conference, Zurich, Switzerland, 2013.

14. L. B. Prasad, B. Tyagi, H. O. Gupta, "Modelling and simulation for optimal control of nonlinear inverted pendulum dynamical system using PID controller and LQR", Asia Modelling Symposium, Bali, Indonesia, 2012.

15. L. B. Prasad, B. Tyagi, H. O. Gupta, "Optimal control of nonlinear inverted pendulum system using PID controller and LQR: Performance analysis without and with disturbance input", International Journal of Automation and Computing, vol. 11, no. 6, pp. 661-670 2014.

16. W. Yu, J. Rosen, "Neural PID control of robot manipulators with application to an upper limb exoskeleton", IEEE Trans Cybern, vol. 43, no. 2, pp. 673-684 2013.

17. A. Kumar, V. Kumar, "Evolving and interval type-2 fuzzy PID controller for the redundant robotic manipulator", Expert Systems with Applications, vol. 73, pp. 161-177, 2017.

18. Y. Su, C. Zheng, "PID control for global finite-time regulation of robotic manipulators," International Journal of Systems Science, vol. 48, no. 3, pp. 547-558, 2017.

19. M. A. Khosravi, H. D. Taghirad, "Robust PID control of fully-constrained cable driven parallel robots", Mechatronics, vol. 24, no. 2, pp. 87-97, 2014.

20. K. Ou, Y. Wang, Z. Li, Y. Shen, D. Xuan, "Feedforward fuzzy-PID control for air flow regulation of PEM fuel cell system," International Journal of Hydrogen Energy, vol. 40, no. 35, pp. 11686-11695, 2015.

21. H. Tang, Y. Li, "Feedforward nonlinear PID control of a novel micromanipulator using Presiach hysteresis compensator," Robotics and Computer-Integrated Manufacturing, vol. 34, pp. 124-132, 2015.

22. M. Taherkhorsandi, M. J. Mahmoodabadi, M. Talebipour, K. K. Castillo-Villar, "Pareto design of an adaptive robust hybrid of PID and sliding control for a biped robot via genetic algorithm optimization", Nonlinear Dynamics, vol. 79, pp. 251-263, 2015.

23. Z. Civelek, M. Lüy, E. Çam, N. Barış̧̧ı, "Control of pitch angle of wind turbine by fuzzy pid controller", Intelligent Automation \& Soft Computing, vol. 22, no. 3, pp. 463-471, 2016.

24. J. Song, W. Cheng, Z. Xu, S. Yuan, M. Liu, "Study on PID temperature control performance of a novel PTC material with room tempera- ture Curie point", International Journal of Heat and Mass Transfer, vol. 95, pp. 1038-1046, 2016.

25. A. M. Simonovic, N. N. Jovanovic, N. S. Lukic, N. D. Zoric, S. N. Stupar, S. S. Ilic, "Experimental studies on active vibration control of smart plate using a modified PID controller with optimal orientation of piezoelectric actuator," Journal of Vibration and Control, vol. 22, no. 1, pp. 2619-2631, 2016.

26. R. Rout, B. Subudhi, "Inverse optimal self-tuning PID control design for an autonomous underwater vehicle", International Journal of Systems Science, vol. 48, no. 2, pp. 367-375, 2017.

27. K. J. Aström, T. Hagglund, C. C. Hang, W. K. Ho, "Automatic tuning and adaptation for PID controllers - a survey," Control Engineering Practice, vol. 1, no. 4, pp. 699-714, 1993.

28. P. Cominos, N. Munro, "PID controllers: recent tuning methods and design to specification," Control Theory and Applications, vol. 149, no. 1, pp. 46-53, 2002.

29. K. Li, "PID tuning for optimal closed-loop performance with specified gain and phase margins", IEEE Transactions on Control, Systems Technology, vol. 21, no. 3, pp. 1024-1030, 2013.

30. B. B. Alagoz, A. Ates, C. Yeroglu, "Auto-tuning of PID controller according to fractional-order reference model approximation for DC rotor control", Mechatronics, vol. 23, pp. 789-797, 2013.

31. A. T. Azar, F. E. Serrano, "Robust IMC-PID tuning for cascade control systems with gain and phase margin specifications", Neural Computing and Applications, vol. 25, pp. 983-995, 2014.

32. C. Y. Jin, H. R. Kyung, S. W. Sung, J. Lee, I. B. Lee, "PID auto-tuning using new model reduction method and explicit PID tuning rule for a fractional order plus time delay model," Journal of Process Control, vol. 24, pp. 113-128, 2014.

33. Ş. Yavuz, L. Malgaca, H. Karagülle, "Analysis of active vibration control of multi-degree-of-freedom flexible systems by Newmark method", Simulation, Modelling and Practice Theory, vol. 69, pp. 136-148, 2016.

34. S. Iplikci, "A comparative study on a novel model-based PID tuning and control mechanism for nonlinear systems", International Journal of Robust and Nonlinear Control, vol. 20, no. 13, pp. 1483-1501, 2010.

35. J. Nocedal, S. J. Wright, Numerical Optimization, Springer: New York, 1999.

36. P. Venkatamaran, Applied Optimization with MATLAB Programming, Wiley-Interscience: New York, 2002.

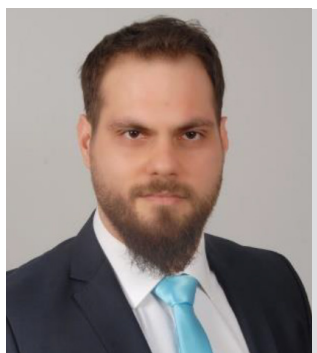

Barış Bıdıklı was born in İzmir, Turkey on September 14th, 1989. He received his BSc, MSc and PhD degrees in Electrical \& Electronics Engineering from Pamukkale University, Denizli, Turkey in 2011, in Electrical \& Electronics Engineering from İzmir Institute of Technology, İzmir, Turkey in 2013 and 2016, respectively. He is currently an Assist. Prof. Dr. at the department of Mechatronics Engineering, Faculty of Engineering and Architecture, Izmir Katip Çelebi University, İzmir, Turkey. His research interests include observer design, adaptive and robust control of uncertain nonlinear dynamical systems, partial state feedback and output feedback control techniques, nonlinear control techniques for mechatronic systems and dynamic simulations of mechatronic systems. 